\title{
IL-17A-producing $T$ cells and associated cytokines are involved in the progression of gastric cancer
}

\author{
FENGYUN ZHONG ${ }^{1 *}$, DAWEI CUI ${ }^{2 *}, \mathrm{HONG} \mathrm{TAO}^{3 *}, \mathrm{HONG}^{1}{ }^{1}$ and CHUNGEN XING ${ }^{1}$ \\ ${ }^{1}$ Department of General Surgery, The Second Affiliated Hospital of Soochow University, Suzhou, Jiangsu 215004; \\ ${ }^{2}$ Center of Clinical Laboratory, The First Affiliated Hospital, School of Medicine, Zhejiang University, \\ Hangzhou, Zhejiang 310003 ; ${ }^{3}$ Department of Optometry and Vision Science, \\ Suzhou Health College, Suzhou, Jiangsu 215002, P.R. China
}

Received May 20, 2015; Accepted June 26, 2015

DOI: $10.3892 / o r .2015 .4246$

\begin{abstract}
Interleukin-17A-producing $\mathrm{T}$ cells $\left(\mathrm{IL}-17 \mathrm{~A}^{+} \mathrm{T}\right)$ (IL-17A ${ }^{+}$CD4 ${ }^{+}$Th17, IL-17A ${ }^{+} \mathrm{CD} 8^{+}$Tc17 and IL-17A ${ }^{+} \gamma \delta \mathrm{T} 17$ cells) and associated cytokines (IL-17A, IL-23 and IL-1 $\beta$ ) play crucial roles in inflammation-associated diseases, such as infection, autoimmunity and tumors. Th17 cells promote human gastric cancer (GC), although the source of intracellular IL-17A and the roles of Tc17 and $\gamma \delta \mathrm{T} 17$ cells remain poorly understood. In this study, the frequencies of circulating Th17 and $\gamma \delta T 17$ cells in patients with GC were found to be significantly increased compared to those in healthy donors; however, Tc17 cells were decreased in these patients, and a negative relationship was found between the frequencies of Th17 and Tc17 cells. Moreover, the cytokine IL-17A was found to be produced mainly by Th17 cells in human peripheral blood. Similarly, serum cytokine levels and relative mRNA expression levels of IL-17A, IL-23 and IL-1 $\beta$ were significantly increased in patients with GC, and the frequency of Th17 cells was closely associated with serum IL-17A concentrations in patients with GC. Additionally, Th17 cells and associated cytokines were present at significantly different levels during the progression and metastasis of GC, as were Tc17 and $\gamma \delta \mathrm{T} 17$ cells. Taken together, these findings suggest that IL-17A ${ }^{+}$ $\mathrm{T}$ cells and associated cytokines might play crucial roles in human GC progression and metastasis and thus represent potential targets for treatment.
\end{abstract}

Correspondence to: Professor Chungen Xing, Department of General Surgery, The Second Affiliated Hospital of Soochow University, 1055 Sanxiang Road, Suzhou, Jiangsu 215004, P.R. China E-mail: xingcg@126.com

*Contributed equally

Key words: interleukin-17, gastric cancer, Th17 cells, Tc17 cells, $\gamma \delta \mathrm{T} 17$ cells

\section{Introduction}

Gastric cancer (GC) is one of the most common and fatal malignancies worldwide. According to Globocan 2012, GC is considered the fifth most common malignancy after lung, breast, colorectum and prostate malignancies (1). It is estimated that $>70 \%$ of GC cases occur in developing countries such as China, Japan and Korea $(1,2)$. Additionally, GC is associated with a high mortality case and is the third leading cause of cancer-related death; the fatality rate is $\sim 75 \%$ of total GC cases worldwide, representing an important public health burden $(1,3)$.

Chronic inflammation-related carcinomas are closely associated with chronic infections, chemical and physical factors, and autoimmune and inflammatory responses of uncertain etiology (4-8). The development, progression and metastasis of GC are closely associated with chronic inflammation associated with Helicobacter pylori infection, the host immune system and other agents (9-12). Inflammation was first linked to cancer by Rudolf Virchow in 1863 (4). Persistent inflammation can induce the formation of cancer cells, and inflammatory cells, cytokines and chemokines in the tumor microenvironment can contribute to cancer growth, invasion and metastasis and to the transformation to an immunosuppressive environment in malignant disease $(7,8,13)$.

Recently, CD4 ${ }^{+} \mathrm{T}$ helper (Th17) cells, a new subtype of $\mathrm{T}$ cells and its associated inflammatory cytokines, such as interleukin-17A (IL-17A), IL-23 and IL-1 $\beta$, play critical roles in the development and metastasis of various tumors including $\mathrm{GC}$, colorectal cancer (CRC), hepatocellular carcinoma (HCC) and ovarian cancer (14-19). IL-17A production has been found not only in Th17 cells but also in other immune cells, including mast cells, IL-17A-secreting $\gamma \delta \mathrm{T}(\gamma \delta \mathrm{T} 17)$ cells, IL-17A-secreting $\mathrm{CD}^{+} \mathrm{T}(\mathrm{Tc} 17)$ cells and NKT cells (20-24). These immune cells have also been suggested to predominately produce IL-17A in other tumor types or in the same tumor type in different species (25-28). Mast cells but not $\mathrm{T}$ cells or macrophages mainly secrete IL-17A in esophageal squamous cell carcinoma tissues and in GC tissue $(21,25)$. Additionally, intracellular IL-17A is produced predominantly by Tc17 cells in human HCC tissue but is mainly produced by Th17 cells in the peripheral blood 
of human HCC patients $(26,27)$. Although $\gamma \delta T 17$ cells are the main cellular source of IL-17A in human CRC, it has been suggested that IL-17A is mainly secreted by Th17 cells in murine colon cancer models $(16,28)$. Similarly, mast cells are the main source of IL-17A in human GC tissue (21); however, the source of IL-17A-producing cells in peripheral blood from human GC patients remains poorly defined. Recent studies have shown that increased percentages of Th17 cells, Tc17 cells and their associated cytokines (such as IL-17A, IL-23, IL-6 and IL-22) promote GC development and progression $(15,29,30)$. However, the roles of $\gamma \delta \mathrm{T} 17$ cells have not been elucidated in human and mouse GC.

Here, we found that the frequencies of Th17 and $\gamma \delta T 17$ cells were significantly higher in peripheral blood from patients with GC than in peripheral blood from healthy donors (HDs); however, Tc17 cell numbers were lower in patients with GC. Moreover, Th17 cells were found to be the main source of intracellular IL-17A-secreting cells, and the second most important source was Tc17 cells; $\gamma \delta \mathrm{T} 17$ cells represented a weak source of these cells in peripheral blood obtained from patients with GC and HDs. In addition, the expression levels of IL-17A and its associated cytokines (such as IL-23 and IL-1 $\beta$ ) in serum and the relative mRNA levels in human peripheral blood mononuclear cells (PBMCs) obtained from patients with GC were markedly higher than in those obtained from HDs, and these IL-17A-producing T cells and associated cytokines were significantly associated with partial clinical pathological characteristics of the patients with GC. Finally, we speculated that Th17 cells, Tc17 cells, $\gamma \delta \mathrm{T} 17$ and IL-17A-associated cytokines may be involved in the progression and metastasis of GC in patients.

\section{Materials and methods}

Patients and specimens. The present study included 47 patients (32 males and 15 females) with GC who underwent surgical operations at the Second Affiliated Hospital, Soochow University from March to October, 2014. The patients did not receive anticancer treatment (such as chemotherapy and radiotherapy) before surgery. Patients with autoimmune diseases or viral infections were excluded from the study. The clinical characteristics of the studied 47 patients with GC and 35 HDs are summarized in Table I. Tumor stage was identified according to the International Union Against Cancer TNM Classification for GC. Tumor tissues were obtained from resected GCs and embedded in paraffin. Serum and peripheral blood specimens were collected from these patients before surgery and from HDs. In addition, written informed consent was obtained from all individuals in accordance with the Declaration of Helsinki (1964); the local Medical Ethics Committee of the Second Affiliated Hospital of Soochow University approved the study.

Cell isolation and flow cytometric analysis. Human PBMCs were isolated from the fresh peripheral blood of all individuals using Ficoll-Hypaque density gradient centrifugation and transferred to sterile tubes containing RPMI-1640 medium supplemented with $10 \%$ fetal bovine serum, $2 \mathrm{mM}$ glutamine, $100 \mathrm{U} / \mathrm{ml}$ penicillin and $100 \mu \mathrm{g} / \mathrm{ml}$ streptomycin (Invitrogen, Carlsbad, CA, USA). The PBMCs
Table I. Clinical features of the patients with GC and healthy donors.

\begin{tabular}{lcc}
\hline Features & GC patients & Healthy donors \\
\hline Gender & & \\
Male & 32 & 21 \\
Female & 15 & 12 \\
Age (years) & & \\
Median & 59 & 57 \\
Range & $32-76$ & $30-75$ \\
Histologic grade & & \\
Well/moderate & 29 & \\
Poor & 18 &
\end{tabular}

Tumor stage

T1 6

T2 24

T3 13

T4 4

Lymphoid nodal status

No 27

N1 13

$\mathrm{N} 2 \quad 5$

N3 2

Distant metastasis status

M0

44

M1

3

TNM stage

I

II

22

6

13

IV 6

The stages of tumors were identified according to International Union Against Cancer TNM Classification for gastric cancer.

(1x10\%cells) were stimulated for $5 \mathrm{~h}$ with $50 \mathrm{ng} / \mathrm{ml}$ phorbol 12-myristate 13-acetate (PMA), $1 \mu \mathrm{g} / \mathrm{ml}$ ionomycin and $500 \mathrm{ng} / \mathrm{ml}$ monensin (eBscience, San Diego, CA, USA) in 24-well plates. Subsequently, the cells were harvested and washed twice in phosphate-buffered saline (PBS). When analyzing Th17, Tc17 and $\gamma \delta \mathrm{T} 17$ cells, the cells were stained with phycoerythrin (PE)-conjugated anti-human CD3, fluorescein isothiocyanate (FITC)-conjugated anti-human $\gamma \delta$ TCR, allophycocyanin (APC)-conjugated anti-human CD8 and Pacific Blue-conjugated anti-human CD4 antibodies at $4^{\circ} \mathrm{C}$ for $30 \mathrm{~min}$. After surface staining, the cells were incubated with PerCP-Cy5.5-conjugated anti-human IL-17 antibody after fixation and permeabilization according to the manufacturer's protocols. Isotype-matched antibody controls were used in all procedures. The stained cells were analyzed using a BD FACSVerse flow cytometer (FCM) and FlowJo software, version 7.6.5 (TreeStar, San Carlos, CA, USA). All antibodies were obtained from Biolegend (San Diego, CA, USA). 


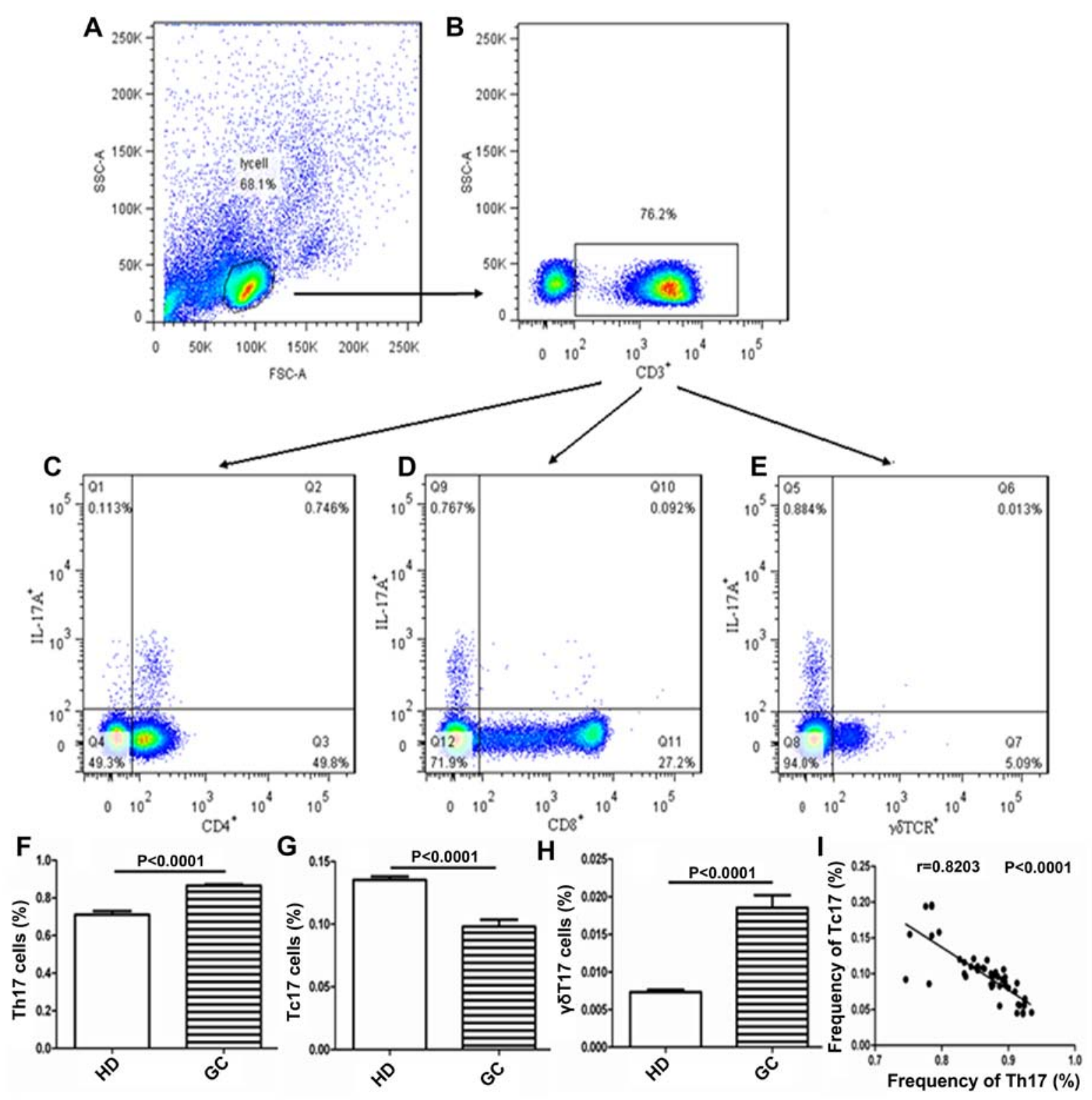

Figure 1. The frequencies of circulating Th17, Tc17 and $\gamma \delta \mathrm{T} 17$ cells in patients with GC. Peripheral blood mononuclear cells (PBMCs) obtained from patients with GC and HDs were stained with labeled antibodies and analyzed using flow cytometry as described in Materials and methods. The cells were gated

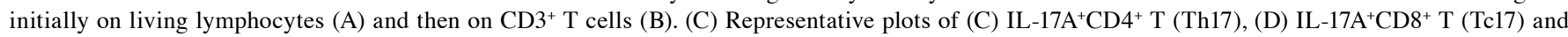

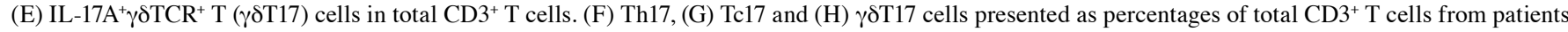
with GC and HDs. (I) Correlation between the frequency of Th17 cells and Tc17 cells in patients with GC. The data shown represent means \pm standard deviation. $\mathrm{P}<0.05$ was considered to represent statistical significance.

Analysis of serum cytokines. Serum cytokines were tested in all individuals using the Bio-Plex Pro Human Th17 Cytokine Panel kit (Bio-Rad Laboratories, Shanghai, China). The cytokines comprised IL-1 $\beta$, IL-17A and IL-23. Multiplex cytokine analysis was performed according to the manufacturer's protocols. Briefly, $50 \mu \mathrm{l}$ of diluted (1:1) beads that were coated with anti-human antibodies raised against the tested cytokine antigen were mixed with $150 \mu \mathrm{l}$ of each diluted sample $(50 \mu \mathrm{l}$ of serum, standards or controls were diluted with $100 \mu \mathrm{l}$ of dilution buffer) and then incubated for $1 \mathrm{~h}$ at room temperature with vigorous shaking. After washing the plate three times, $25 \mu 1$ of biotinylated detection antibodies was added and incubated for $30 \mathrm{~min}$. After a wash cycle, $50 \mu \mathrm{l}$ streptavidin (SA)-PE was added to the beads, and the mixture was incubated for $10 \mathrm{~min}$. After washing the plate three times, the bead mixtures were resuspended in $125 \mu \mathrm{l}$ assay buffer and measured using a Bio-Plex 200 system (Bio-Rad Laboratories, Inc., Munich, Germany). All samples were detected in triplicate. The data were analyzed using BioPlex Manager 6.1 software (Bio-Rad Laboratories, Inc.)

RNA isolation and quantitative real-time PCR ( $q R T-P C R)$. To analyze the mRNA expression of IL-1 $\beta$, IL-17A and IL-23, total RNA was extracted from human PBMCs using TRIzol (Invitrogen) after stimulating the cells for $5 \mathrm{~h}$ with $50 \mathrm{ng} / \mathrm{ml}$ phorbol 12-myristate 13-acetate (PMA) and $1 \mu \mathrm{g} / \mathrm{ml}$ ionomycin. cDNA was synthesized using reverse transcription reagent kits (Takara, Dalian, China) according to the manufacturer's instructions. Real-time PCR was performed in triplicate using the QuantiFast ${ }^{\mathrm{TM}}$ SYBR-Green PCR kit (Qiagen, Hilden, 
Germany) and an ABI 7500 analysis system (Applied Biosystems, Foster, CA, USA). The amplification conditions were as follows: $5 \mathrm{~min}$ at $95^{\circ} \mathrm{C}$ (denaturation); then, 40 cycles of $95^{\circ} \mathrm{C}$ for $10 \mathrm{sec}$ and $60^{\circ} \mathrm{C}$ for $30 \mathrm{sec}$; the fluorescence was recorded at $60^{\circ} \mathrm{C}$. The primer sequences used were as follows: IL-1 $\beta$ forward, 5'-CCACAGACCTTCCAGGAGA ATG-3' and IL-1 $\beta$ reverse, 5'-GTGCAGTTCAGTGATCGTA CAGG-3'; IL-17A forward, 5'-CGGACTGTGATGGTCAAC CTGA-3' and IL-17A reverse, 5'-GCACTTTGCCTCCCAGA TCACA-3'; IL-23p19 forward, 5'-GAGCCTTCTCTGCTCCCTGATA-3' and IL-23p19 reverse, 5'-GACTGAGGCTTGGAATCTG CTG-3'; $\beta$-actin forward, 5'-TGGAATCCTGTGGCATCCA TGAAAC- 3 ' and $\beta$-actin reverse, 5'-TAAAACGCAGCTCA GTAACAGTCCG-3'. The data were analyzed using ABI 7500 software (Applied Biosystems).

Statistical analysis. One-way ANOVA analysis was performed to confirm the statistical significance of differences among the groups. Data are presented as the means \pm standard deviation (SD). Between-group comparisons were performed using Student's t-test. For non-parametric data, the Mann-Whitney $\mathrm{U}$ test was performed between the two studied groups. Correlations between variables were determined using Spearman's correlation coefficient. $\mathrm{P}<0.05$ was considered to indicate a statistically significant difference. The data were analyzed using GraphPad Prism 5 software (GraphPad Software, Inc., San Diego, CA, USA).

\section{Results}

Clinical characteristics of the patients with GC. The clinical characteristics of the patients with GC are presented in Table I. Forty-seven patients, including 32 males and 15 females, were recruited in this study. The age of the patients ranged from 32 to 76 years. Twenty-nine patients presented with well/moderate histology, whereas 18 presented with severe histology. Twenty-seven patients had no lymphoid nodal metastasis, although 13 were classified as N1, 5 were classified as $\mathrm{N} 2$ and 2 were classified as N3. Moreover, most patients $(93.6 \%, 44 / 47)$ had no distant metastasis, but 3 were classified as M1. Additionally, there were $63.8 \%$ (30) cases of tumor stage $\mathrm{T} 1+\mathrm{T} 2$ and 17 cases of stage $\mathrm{T} 3+\mathrm{T} 4$ according to the 7 th edition of the American Joint of Committee on Cancer. Of the cases with GC, 22, 6, 13 and 6 were classified as stages I, II, III and IV, respectively according to the TNM classification for GC.

Frequency of circulating Th17, Tc17 and $\gamma \delta T 17$ cells in peripheral blood from patients with $G C$. The frequencies of circulating Th17, Tc17 and $\gamma \delta \mathrm{T} 17$ cells in gated $\mathrm{CD}^{+} \mathrm{T}$ cells in the PBMCs obtained from patients with $\mathrm{GC}$ were detected to explore their roles in the patients with GC (Fig. 1A-I). The frequencies of circulating Th17 and $\gamma \delta T 17$ cells were significantly higher in patients with GC than in the HDs (Fig. $1 \mathrm{~F}$ and $\mathrm{H}$ ), although the frequency of circulating Tc17 cells was notably lower in patients with GC than in the HDs (Fig. 1G). Additionally, the frequency of Th17 cells was negatively associated with that of Tc17 cells in the patients with GC (Fig. 1I). Further analysis indicated that there was no significant difference in regards to the frequency of Th17 cells
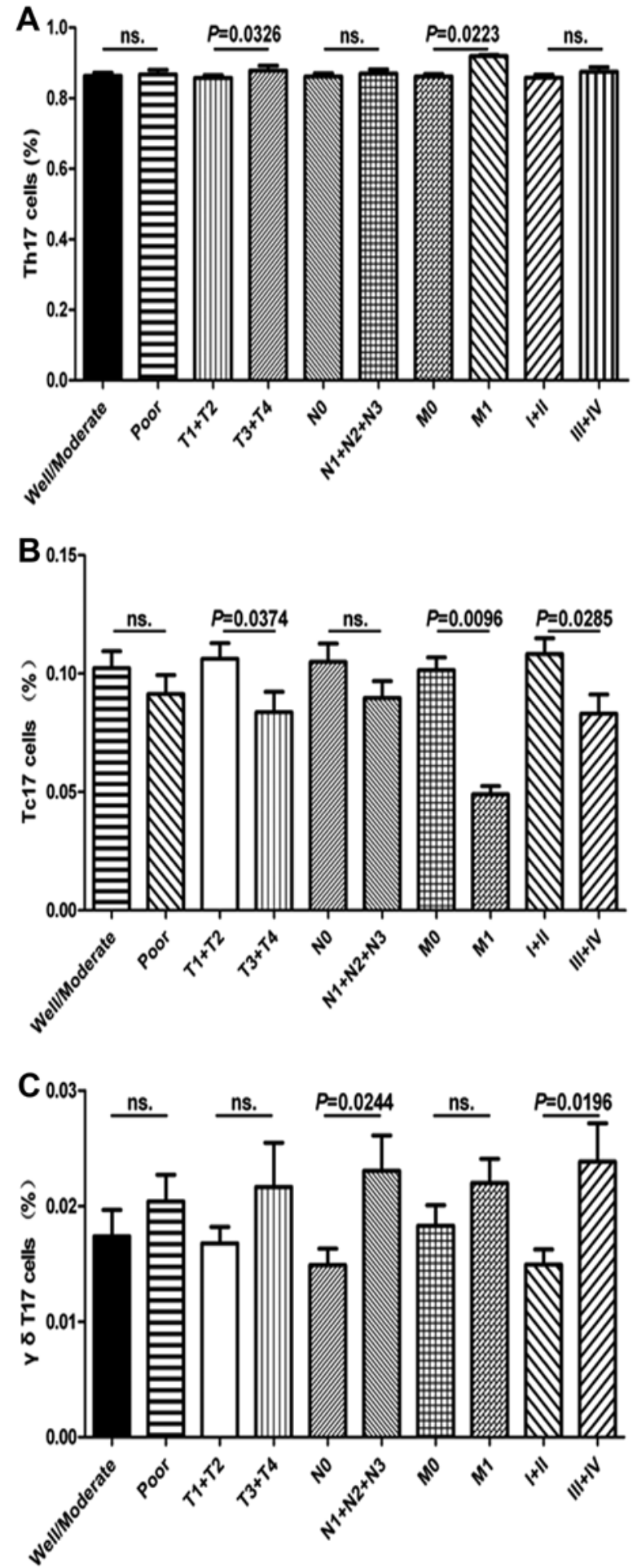

Figure 2. Analysis of the correlation of circulating cells in patients with GC with different tumor characteristics. (A) The percentage of Th17 cells was compared in patients according to tumor differentiation (good/moderate vs. poor), depth of tumor invasion (T1+T2 vs. T3+T4), lymphoid nodal metastasis (N0 vs. $\mathrm{N} 1+\mathrm{N} 2+\mathrm{N} 3$ ), distant metastasis (M0 vs. M1) and TNM stage (I+II and III+IV). (B) The percentage of Tc17 cells was compared to these same parameters. (C) The percentage of $\gamma \delta T 17$ cells was compared to these same parameters. The data shown represent means \pm standard deviation. $\mathrm{P}<0.05$ was considered to represent statistical significance; ns., no significant difference.

in regards to clinicopathologic features such as tumor differentiation (well/moderate vs. poor), TNM stage (I+II and III+IV) and lymphoid nodal metastasis (N0 vs. $\mathrm{N} 1+\mathrm{N} 2+\mathrm{N} 3$ ); however, 

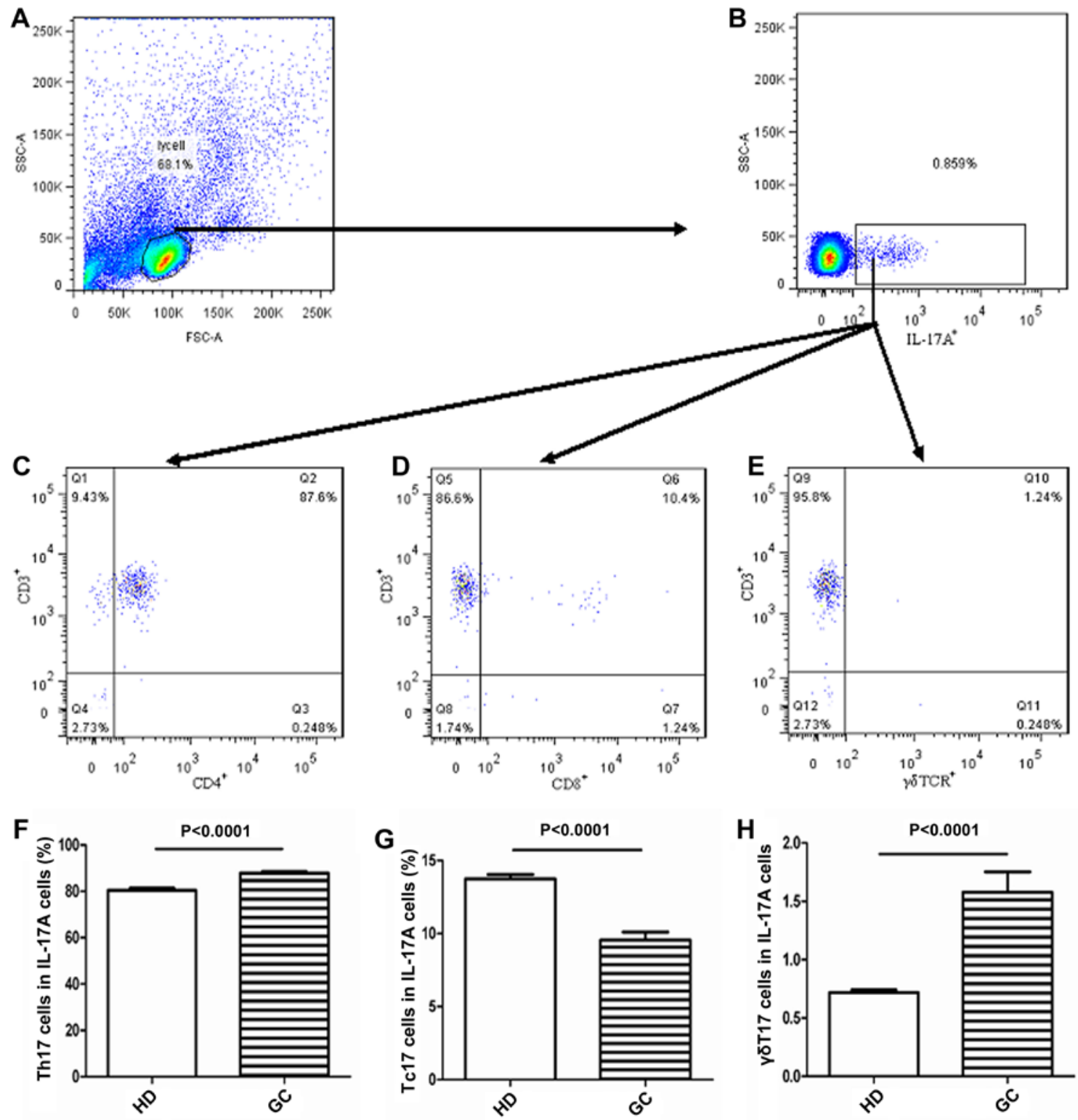

Figure 3. Percentages of Th17, Tc17 and $\gamma \delta T 17$ cells in IL-17A cells. Peripheral blood mononuclear cells (PBMCs) obtained from patients with GC and from HDs were stained with labeled antibodies and analyzed using flow cytometry as described in Materials and methods. The cells were gated initially on living

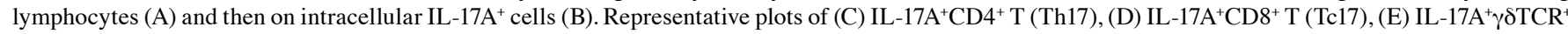

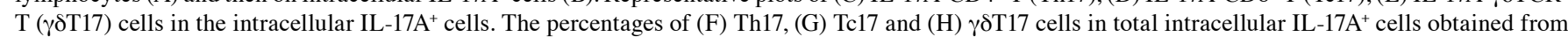
patients with GC and from HDs. The data shown represent means \pm standard deviation. $\mathrm{P}<0.05$ was considered to represent statistical significance.

a significant association with tumor invasion (T1+T2 vs. T3+T4) and distant metastasis (M0 vs. M1) was observed in patients with GC (Fig. 2A). The percentage of $\gamma \delta T 17$ cells in patients with GC with lymphoid nodal metastasis $(\mathrm{N} 1+\mathrm{N} 2+\mathrm{N} 3)$ was significantly higher than that in patients with $\mathrm{GC}$ with no lymphoid nodal metastasis (N0), similar to TNM stage (I+II and III+IV); however, no significant difference was found between other clinical characteristics and the percentage of $\gamma \delta \mathrm{T} 17$ cells, respectively (Fig. 2C). The percentage of Tc17 cells in patients with III+IV stage GC was significantly lower than that in patients with I+II stage GC; similar findings were observed for some clinical features, such as distant metastasis (M0 vs. M1) and depth of tumor invasion (T1+T2 vs. T3+T4), but not for lymphoid nodal metastasis $(\mathrm{N} 1+\mathrm{N} 2+\mathrm{N} 3)$ or tumor differentiation (good/moderate vs. poor) (Fig. 2B), respectively. These findings indicate that the patients with GC generally had higher populations of circulating Th17 and $\gamma \delta \mathrm{T} 17$ cells and a decreased percentage of circulating Tc17 cells in peripheral blood, which were involved in the progression of GC in the patients.

The principal source of intracellular IL-17A cells in peripheral blood. Since circulating Th17, Tc17 and $\gamma \delta T 17$ cells play important roles in patients with GC, we determined the principal source of intracellular IL-17A cytokines in peripheral blood obtained from the patients with GC by measuring the frequencies of circulating Th17, Tc17 and $\gamma \delta T 17$ cells in gated intracellular IL-17A cells obtained from patients with GC using FCM (Fig. 3A-E). The proportion of circulating Th17 cells was highest in intracellular IL-17A cells 

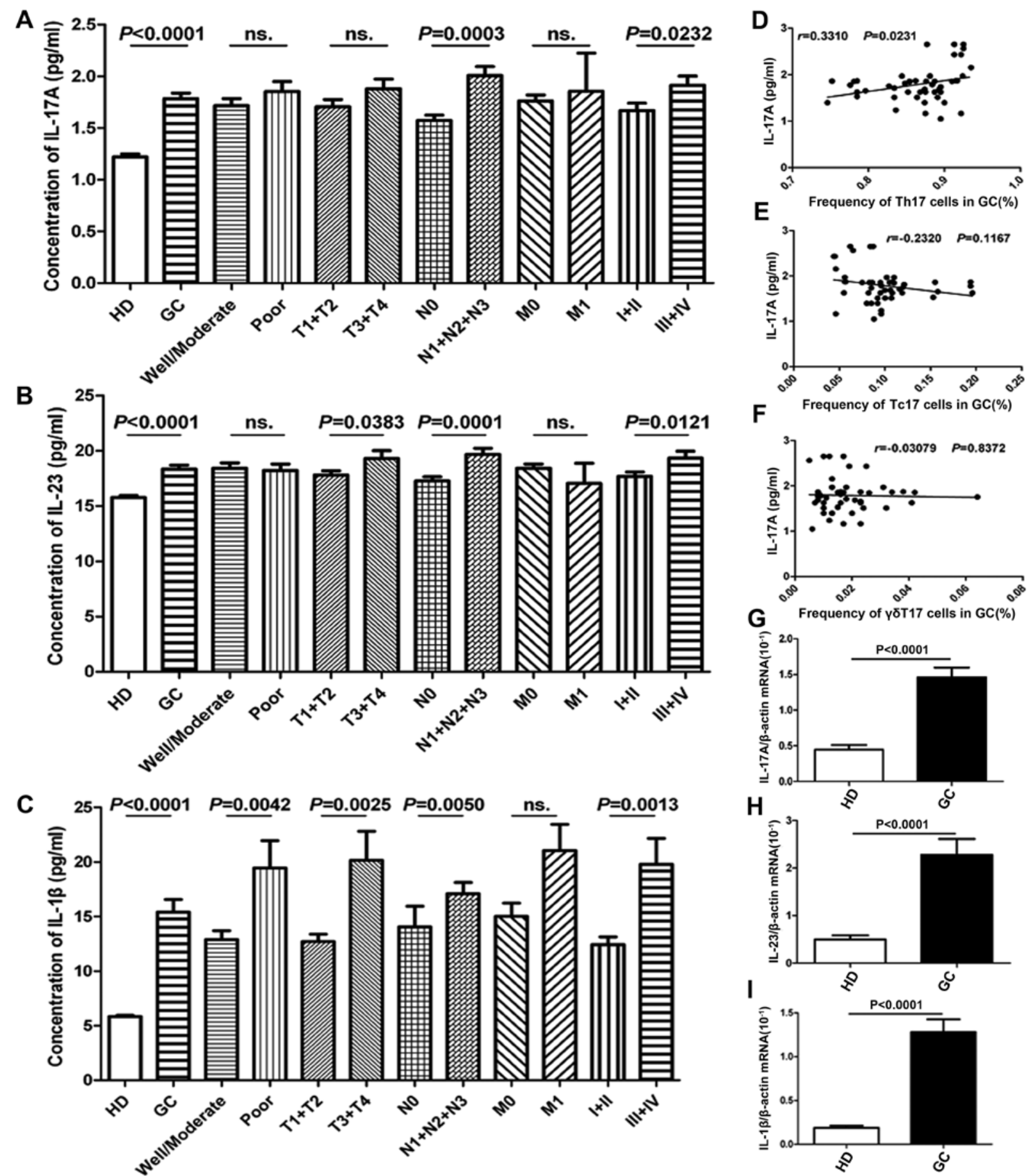

Figure 4. Expression levels of the cytokines IL-17A, IL-23 and IL-1 $\beta$ in serum and PBMCs. Serum (A) IL-17A (B) IL-23 and (C) IL-1 $\beta$ concentrations in HDs and in patients with GC according to different tumor characteristics. Relationships between IL-17A levels and the frequency of circulating (D) Th17, (E) Tc17 and (F) $\gamma \delta$ T17 cells. Relative (G) IL-17A, (H) IL-23 and (I) IL-1 $\beta$ mRNA expression levels in PBMCs. P $<0.05$ was considered to represent statistical significance; ns., no significant difference.

of peripheral blood cells from patients with GC or the HDs (Fig. 3C). The second-largest population of intracellular IL-17A cells were circulating Tc17 cells (Fig. 3D) and the lowest population of circulating Tc17 cells was $\gamma \delta T 17$ cells (Fig. 3E). Moreover, the percentages of intracellular IL-17A cells represented by circulating Th17 and $\gamma \delta T 17$ cells in peripheral blood were significantly higher in patients with GC than in HDs (Fig. 3F and H). In contrast, the percentage of intracellular IL-17A cells represented by circulating Tc17 obtained from patients with GC was obviously lower than that in the HDs (Fig. 3G). These data imply that circulating Th17 cells represent the main source of intracellular IL-17A cytokine in peripheral blood and that the percentages of circulating Th17 and $\gamma \delta \mathrm{T} 17$ cells were notably increased in patients with GC than in the HDs.

Expression of IL-17A and associated cytokines in patients with GC. Since circulating Th17, Tc17 and $\gamma \delta T 17$ cells were 
found to be an important source of intracellular IL-17A cytokines in peripheral blood, we further detected the expression levels of IL-17A and its associated cytokines (IL-23 and IL-1 $\beta$ ) in serum and PBMCs obtained from patients with $\mathrm{GC}$ and from HDs (Fig. 4A-G). The concentrations of the cytokines IL-17A, IL-23 and IL-1 $\beta$ were significantly higher in sera obtained from patients with GC than in sera obtained from the HDs (Fig. 4A-C). Moreover, significant associations were found between IL-17A levels and various clinical pathological characteristics including lymphoid nodal metastasis (N0 vs. $\mathrm{N} 1+\mathrm{N} 2+\mathrm{N} 3)$ and TNM stage (I+II and III+IV), respectively. However, no significant association between IL-17A concentration and tumor histopathology (well/moderate vs. poor), depth of tumor invasion (T1+T2 vs. T3+T4) or distant metastasis (M0 vs. M1) was observed in the patients with GC (Fig. 4A). Similar findings regarding IL-23 and IL-1 $\beta$ concentrations were observed in patients with GC (Fig. 4B and C). Additionally, serum IL-23 and IL-1 $\beta$ levels were markedly different between patients with GC at stages T1+T2 and T3+T4 and IL-1 $\beta$ levels were also significantly different between good/moderate and poor tumor histopathology (Fig. 4B and C). Moreover, serum IL-17A concentrations were markedly associated with Th17 cell frequency in patients with GC but not with Tc17 and $\gamma \delta \mathrm{T} 17$ cell frequencies (Fig. 4D-F). In addition, the mRNA expression levels of IL-1 $\beta$, IL-17A and IL-23 were notably higher in PBMCs in patients with GC than in those of the HDs (Fig. 4G-I).

\section{Discussion}

In the present study, we found that the frequencies of circulating Th17 and $\gamma \delta \mathrm{T} 17$ cells were significantly higher in peripheral blood obtained from the patients with GC than in the HDs; however, the percentage of Tc17 cells was lower. Moreover, the expression levels of the associated cytokines, such as IL-17A, IL-23 and IL-1 $\beta$ in serum and PBMCs obtained from patients with GC were also significantly higher than those of the HDs, and Th17, Tc17 and $\gamma \delta T 17$ cells and cytokines were partly associated with clinicopathologic characteristics such as cancer invasion, metastasis and TNM stage. Furthermore, the main source of intracellular IL-17A cytokine was Th17 cells, the second-most important source was Tc17 cells and $\gamma \delta T 17$ cells also acted as a source of intracellular IL-17A cytokine in peripheral blood obtained from patients with GC. Taken together, these findings suggest that Th17, Tc17 and $\gamma \delta T 17$ cells might play crucial roles in human GC development and metastasis.

Th17 cells play pivotal roles in inflammation and in autoimmune diseases of humans and mice, thus exacerbating the severity of diseases; they also act in host defense against pathogens (31-33). Recently, accumulating evidence has suggested that Th17 cells exhibit complex and controversial roles in the development, progression and metastasis of tumors $(30,34,35)$. An increased prevalence of Th17 cells in peripheral blood, tumor tissue or tumor-draining lymph nodes contributes to the development, progression and/or metastasis of human cancers, such as gastric carcinoma, CRC, HCC and pancreatic cancer $(16,26,30)$. In contrast, a high frequency of tumor-infiltrating Th17 cells is associated with improved survival in ovarian and lung carcinoma patients and with slower progression in patients with prostate cancer (34-36). Similarly, increased numbers of Th17 cells in the tumor environment improve survival in mouse models of pancreatic and melanoma cancer $(37,38)$. Although the frequency of Th17 cells was obviously higher in peripheral blood obtained from patients with GC, it was not directly confirmed that these cells were the main source of IL-17 in human peripheral blood.

Our results demonstrated that the frequency of circulating Th17 cells was significantly higher in PBMCs obtained from patients with GC than in that obtained from HDs, consistent with previous studies $(15,21,30)$. Moreover, it was clear that intracellular IL-17A cytokines were mainly produced by Th17 cells obtained from the PBMCs of patients with GC and HDs. Additionally, the high percentage of circulating Th17 cells was individually associated with depth of tumor invasion (T1+T2 vs. T3+T4) and distant metastasis (M0 vs. M1) but not with other factors such as TNM stage (I+II and $\mathrm{III}+\mathrm{IV}$ ) and lymphoid nodal metastasis (N0 vs. N1+N2+N3). These findings suggest that Th17 cells partly participate in the progression and metastasis of human GC, a finding that is partially consistent with previous reports $(15,30)$. The discrepancies found might be due to the numbers of recruited patients, age, lifestyle and other causative agents, such as infections, chemical and physical factors and other factors of uncertain etiology.

Similar to Th17 cells, Tc17 cells that are defined by a signature of IL-17A-secreting $\mathrm{CD}^{+} \mathrm{T}$ cells play a critical role in various diseases, such as those related to infection and autoimmunity (39-42). For example, in rhesus macaques with pathogenic simian immunodeficiency virus (SIV) infection, Tc17 cells regulate the disease progression and contribute to the disease outcome (39). Additionally, Tc17 cells can protect mice against lethal influenza infection, which is accompanied by an early enhanced influx of neutrophils into the lung (40). Conversely, Tc17 cells, but not Th17 cells, notably caused severe autoimmune colitis in a mouse model (41). In an animal model of multiple sclerosis (MS), the numbers of IL- $17^{+} \mathrm{CD}^{+} \mathrm{T}$ ( $\left.\mathrm{Tc} 17\right)$ cells were found to be significantly higher in active regions of MS lesions than in inactive areas and controls, suggesting that Tc17 cells are associated with active disease in MS (42). However, the clinical prevalence and role of Tc17 cells remain elusive in human and mouse tumors (43).

Recent studies indicate that Tc17 cells have important roles in the development and metastasis of tumors (44-48). Elevated proportions of Tc17 cells in patients with cervical cancers are associated with cancer progression that is accompanied by increased infiltrations of Th17 cells as well as enhanced tumor vasculogenesis and metastasis (44). Similar results were also observed in patients with endometrial carcinoma (45). In contrast, adoptive transfer of Tc17 cells was found to mediate effective antitumor immunity against an established murine melanoma model, and this depended on the effects of IFN- $\gamma$ in the tumor environment $(46,47)$.

A high proportion of Th17 and a low proportion of Tc17 cells are observed in peripheral blood obtained from patients with thyroid tumors, and these proportions are negatively correlated with tumor size, suggesting that Tc17 cells might have an antitumor role in thyroid tumors and that its effective function may not be involved with IL-17 
production (48). Recently, Tc17 cell numbers were found to be elevated in peripheral $\mathrm{CD}^{+} \mathrm{T}$ cells and in tumor tissues obtained from patients with GC (29). However, increased Tc17 cell frequency was not directly shown in PBMCs obtained from patients with GC, and it was unclear whether Tc17 cells were the main source of intracellular IL-17A in patients with GC and in HDs.

Here, a significantly decreased frequency of Tc17 cells was observed in patients with GC and was notably associated with depth of tumor invasion and TNM stage but not with other factors, such as lymphoid nodal metastasis and tumor differentiation. Moreover, Tc17 cells were the second most important source of intracellular IL17A cytokine in the patients with GC and in HDs. Interestingly, a negative association was found between the frequency of Th17 and Tc17 cells in patients with GC, indicating that increased percentage of Th17 cells might negatively regulate $\mathrm{Tc} 17$ cells in patients with GC and that Tc17 cells might exert antitumor activity in patients with GC; however, this notion requires further investigation by studying the role of Tc17 cells in human GC. Taken together, these findings indicate that a low percentage of Tc17 cells might affect the development and progression of GC. The different age, population and causative factors in our patients with GC, together with differences in the study aim, might account for the discrepancies between our results and those described in a previous study (29).

$\gamma \delta T 17$ cells, as innate cells that secrete the IL-17 cytokine, are involved in the pathogenesis of various inflammation-associated diseases in humans and mice $(49,50)$. $\gamma \delta T 17$ cells play an effective and pivotal role in psoriasiform plaque formation in mice and are the primary source of IL-17A cytokine but not Th17 cells (51). Additionally, a high frequency of $\gamma \delta T 17$ cells is found in the brains of mice with experimental autoimmune encephalomyelitis (EAE), and the activation of $\gamma \delta T 17$ cells can promote the expansion of Th17 cells and increase susceptibility to EAE (50). Elevated $\gamma \delta T 17$ cells exacerbate arthritis and are the main source of IL-17 cytokine in mice with collagen-induced arthritis (CIA) (52). However, the role of $\gamma \delta \mathrm{T} 17$ cells is poorly understood in tumors.

Tumor-infiltrating IL-17 promotes the progression of skin carcinoma in mice by inducing angiogenesis and is mainly produced by $\gamma \delta \mathrm{T} 17$ cells in T cell tumor-infiltrating lymphocytes (TILs) but not in Th17 or Tc17 cells (53). Additionally, the frequencies of Th17, Tc17 and $\gamma \delta \mathrm{T} 17$ cells are all clearly elevated in human PBMCs and in the tumor tissues of patients with CRC (16). Moreover, $\gamma \delta T 17$ cells are the main producers of IL-17A and promote the progression of human CRC (16). Dissimilarly, Th17 cells, not $\gamma \delta \mathrm{T} 17$ cells, are considered the main source of IL-17 and were found to improve the progression of a murine colon cancer model (28). Although the frequency of Th17 and/or Tc17 cells was obviously elevated in patients with GC $(15,16,30)$, it has not been clearly proven that $\gamma \delta \mathrm{T} 17$ cells are the main source of IL-17, and the role of $\gamma \delta \mathrm{T} 17$ cells has not yet been elucidated in human GC.

Our results demonstrated that a significantly increased frequency of $\gamma \delta T 17$ cells was observed in patients with GC, and this increase was notably associated with lymphoid nodal metastasis and TNM stage but not with other factors, such as depth of tumor invasion, distant metastasis and tumor differentiation. In addition, $\gamma \delta \mathrm{T} 17$ cells were not an abundant source of intracellular IL-17A cytokine; rather, they were a poorer source than Th17 and Tc17 cells in patients with GC and in HDs. Taken together, these findings indicate that an increased percentage of $\gamma \delta T 17$ cells might contribute to the development and progression of GC. Moreover, human GC represents a possible cause for the low production of intracellular IL-17A from $\gamma \delta T 17$ cells, implying that various tumors might affect the phenotype and distribution of IL-17A-producing $\mathrm{T}$ cells differently and act as the main source of intracellular IL-17A cytokine. We will explore the role of $\gamma \delta T 17$ cells in human GC in future studies.

IL-23 and/or IL-1 $\beta$ cytokines, which are secreted via innate immune cells, such as dendritic cells and macrophages, can contribute to the proliferation and differentiation of Th17, Tc17 or $\gamma \delta T 17$ cells, which promote the production of the IL-17 cytokine (54-56). Increased IL-17, IL-23 and/or IL-1 $\beta$ cytokines, which are accompanied by elevated percentages of Th17, Tc17 or $\gamma \delta \mathrm{T} 17$ cells, play important roles in inflammation-associated diseases, including those related to infection, autoimmunity and tumors $(14,49,50,54)$. Recently, serum concentrations and mRNA expression levels of IL-17A, IL-23 and/or IL-1 $\beta$, which are associated with Th17 and Tc17 cells, have been found to be significantly increased in patients with GC compared to those in healthy individuals and are closely associated with the development of gastric carcinoma $(15,21,29,30)$. IL-23 and IL-1 $\beta$ cytokines, which are produced by tumor-activated monocytes, promote the differentiation of Tc17 cells that contribute to cancer progression in patients with GC (29).

Our results showed that serum IL-17, IL-23 and IL-1 $\beta$ levels were obviously increased in patients with GC, and this increase was associated with stages of GC development, such as lymphoid nodal metastasis and the TNM stage and was partly associated with depth of tumor invasion and distant metastasis; in addition, Th17 cell percentage was positively associated with serum IL-17A concentrations in patients with GC. Additionally, the relative mRNA expression of these cytokines was significantly higher in patients with GC than in HDs, consistent with previous studies $(15,21,30)$. These findings indicate that these increases in the inflammatory cytokines IL-17, IL-23 and IL-1 $\beta$ contribute to the progression and metastasis of human GC and to the proliferation of inflammatory cells, such as Th17 cells. These inflammatory cytokines and associated inflammation-related cells play important roles in the development, progression and metastasis of human GC.

In summary, our results revealed that the frequencies of circulating Th17 and $\gamma \delta \mathrm{T} 17$ cells were significantly higher in patients with GC than in HDs; however, the frequency of Tc17 cells, which were involved in the progression and metastasis of patients with GC, was decreased. Moreover, the intracellular IL-17A cytokine was predominantly produced by Th17 cells in human peripheral blood. Additionally, the expression levels of Th17 cell-associated cytokines, such as IL-17A, IL-23 and IL-1 $\beta$, were significantly elevated in the serum and PBMCs of patients with GC and played important roles in the progression and metastasis in the patients with GC. In future studies, a large number of patients with GC will be studied to determine the roles of Th17, Tc17 and $\gamma \delta T 17$ cells in the pathogenesis of GC. 


\section{Acknowledgements}

This study was supported by the Scientific Research Innovation Projects for Ordinary University Graduate Students, Jiangsu, China (grant no. CXLX12_0842), the Pre-Research Fund of Soochow University (grant no. SDY2012B28) and the Pre-Research Fund of the Second Affiliated Hospital of Soochow University (grant no. SDFEYGJ1301). The manuscript had been edited for English language by American Journal Experts (http://www.aje. com).

\section{References}

1. Ferlay J, Soerjomataram I, Dikshit R, Eser S, Mathers C, Rebelo M, Parkin DM, Forman D and Bray F: Cancer incidence and mortality worldwide: Sources, methods and major patterns in GLOBOCAN 2012. Int J Cancer 136, E359-E386, 2015.

2. Ferlay J, Shin H.R, Bray F, Forman D, Mathers C and Parkin DM: Estimates of worldwide burden of cancer in 2008: GLOBOCAN 2008. Int J Cancer 127, 2893-2917, 2010.

3. McLean MH and El-Omar EM: Genetics of gastric cancer. Nat Rev Gastroenterol Hepatol 11: 664-674, 2014.

4. Balkwill $\mathrm{F}$ and Mantovani A: Inflammation and cancer: Back to Virchow? Lancet 357: 539-545, 2001.

5. Okada F: Inflammation-related carcinogenesis: Current findings in epidemiological trends, causes and mechanisms. Yonago Acta Med 57: 65-72, 2014.

6. De Flora $\mathrm{S}$ and Bonanni P: The prevention of infection-associated cancers. Carcinogenesis 32: 787-795, 2011.

7. Trinchieri G: Cancer and inflammation: An old intuition with rapidly evolving new concepts. Annu Rev Immunol 30: 677-706, 2012.

8. Grivennikov SI, Greten FR and Karin M: Immunity, inflammation and cancer. Cell 140: 883-899, 2010.

9. Correa P: Helicobacter pylori and gastric carcinogenesis. Am J Surg Pathol 19 (Suppl 1): S37-S43, 1995.

10. Yakirevich E and Resnick MB: Pathology of gastric cancer and its precursor lesions. Gastroenterol Clin North Am 42: 261-284, 2013.

11. Piazuelo MB and Correa P: Gastric cáncer: Overview. Colomb Med (Cali) 44: 192-201, 2013.

12. Grabsch HI and Tan P: Gastric cancer pathology and underlying molecular mechanisms. Dig Surg 30: 150-158, 2013.

13. Mantovani A, Allavena P, Sica A and Balkwill F: Cancer-related inflammation. Nature 454: 436-444, 2008.

14. Yang B, Kang H, Fung A, Zhao H, Wang T and Ma D: The role of interleukin 17 in tumour proliferation, angiogenesis, and metastasis. Mediators Inflamm 2014: 623759, 2014.

15. Zhang B, Rong G, Wei H, Zhang M, Bi J, Ma L, Xue X, Wei G, Liu X and Fang G: The prevalence of Th17 cells in patients with gastric cancer. Biochem Biophys Res Commun 374: 533-537, 2008.

16. Wu P, Wu D, Ni C, Ye J, Chen W, Hu G, Wang Z, Wang C, Zhang Z, Xia W, et al: $\gamma \delta \mathrm{T} 17$ cells promote the accumulation and expansion of myeloid-derived suppressor cells in human colorectal cancer. Immunity 40: 785-800, 2014.

17. He D, Li H, Yusuf N, Elmets CA, Li J, Mountz JD and Xu H: IL-17 promotes tumor development through the induction of tumor promoting microenvironments at tumor sites and myeloid-derived suppressor cells. J Immunol 184: 2281-2288, 2010.

18. Miyahara Y, Odunsi K, Chen W, Peng G, Matsuzaki J and Wang RF: Generation and regulation of human CD4 ${ }^{+}$IL-17-producing T cells in ovarian cancer. Proc Natl Acad Sci USA 105: 15505-15510, 2008.

19. Langowski JL, Zhang X, Wu L, Mattson JD, Chen T, Smith K, Basham B, McClanahan T, Kastelein RA and Oft M: IL-23 promotes tumour incidence and growth. Nature 442: 461-465, 2006.

20. Alizadeh D, Katsanis E and Larmonier N: The multifaceted role of Th17 lymphocytes and their associated cytokines in cancer. Clin Dev Immunol 2013: 957878, 2013.
21. Liu X, Jin H, Zhang G, Lin X, Chen C, Sun J, Zhang Y, Zhang Q and Yu J: Intratumor IL-17-positive mast cells are the major source of the IL-17 that is predictive of survival in gastric cancer patients. PLoS One 9: e106834, 2014.

22. Lockhart E, Green AM and Flynn JL: IL-17 production is dominated by gammadelta $\mathrm{T}$ cells rather than CD4 T cells during Mycobacterium tuberculosis infection. J Immunol 177: 4662-4669, 2006.

23. Liu SJ, Tsai JP, Shen CR, Sher YP, Hsieh CL, Yeh YC, Chou AH, Chang SR, Hsiao KN, Yu FW, et al: Induction of a distinct CD8 Tnc17 subset by transforming growth factor-beta and interleukin-6. J Leukoc Biol 82: 354-360, 2007.

24. Yoshiga Y, Goto D, Segawa S, Ohnishi Y, Matsumoto I, Ito S, Tsutsumi A, Taniguchi $M$ and Sumida T: Invariant NKT cells produce IL-17 through IL-23-dependent and -independent pathways with potential modulation of Th17 response in collagen-induced arthritis. Int J Mol Med 22: 369-374, 2008.

25. Wang B, Li L, Liao Y, Li J, Yu X, Zhang Y, Xu J, Rao H, Chen S, Zhang L, et al: Mast cells expressing interleukin 17 in the muscularis propria predict a favorable prognosis in esophageal squamous cell carcinoma. Cancer Immunol Immunother 62: 1575-1585, 2013.

26. Zhang JP, Yan J, Xu J, Pang XH, Chen MS, Li L, Wu C, Li SP and Zheng L: Increased intratumoral IL-17-producing cells correlate with poor survival in hepatocellular carcinoma patients. J Hepatol 50: 980-989, 2009.

27. Kuang DM, Peng C, Zhao Q, Wu Y, Zhu LY, Wang J, Yin XY, Li L and Zheng L: Tumor-activated monocytes promote expansion of IL-17-producing $\mathrm{CD}^{+} \mathrm{T}$ cells in hepatocellular carcinoma patients. J Immunol 185: 1544-1549, 2010.

28. Grivennikov SI, Wang K, Mucida D, Stewart CA, Schnabl B, Jauch D, Taniguchi K, Yu GY, Osterreicher CH, Hung KE, et al: Adenoma-linked barrier defects and microbial products drive IL-23/IL-17-mediated tumour growth. Nature 491: 254-258, 2012.

29. Zhuang Y, Peng LS, Zhao YL, Shi Y, Mao XH, Chen W, Pang KC, Liu XF, Liu T, Zhang JY, et al: CD8(+) T cells that produce interleukin-17 regulate myeloid-derived suppressor cells and are associated with survival time of patients with gastric cancer. Gastroenterology 143: 951-962, 2012.

30. Iida $T$, Iwahashi $M$, Katsuda $M$, Ishida $K$, Nakamori $M$, Nakamura M, Naka T, Ojima T, Ueda K, Hayata K, et al: Tumor-infiltrating CD4 ${ }^{+}$Th17 cells produce IL-17 in tumor microenvironment and promote tumor progression in human gastric cancer. Oncol Rep 25: 1271-1277, 2011.

31. Fouser LA, Wright JF, Dunussi-Joannopoulos K and Collins M: Th17 cytokines and their emerging roles in inflammation and autoimmunity. Immunol Rev 226: 87-102, 2008

32. Wilke CM, Bishop K, Fox D and Zou W: Deciphering the role of Th17 cells in human disease. Trends Immunol 32: 603-611, 2011

33. Muranski P and Restifo NP: Essentials of Th17 cell commitment and plasticity. Blood 121: 2402-2414, 2013.

34. Kryczek I, Banerjee M, Cheng P, Vatan L, Szeliga W, Wei S, Huang E, Finlayson E, Simeone D, Welling TH, et al: Phenotype, distribution, generation, and functional and clinical relevance of Th17 cells in the human tumor environments. Blood 114: 1141-1149, 2009

35. Sfanos KS, Bruno TC, Maris $\mathrm{CH}, \mathrm{Xu} \mathrm{L}$, Thoburn CJ, DeMarzo AM, Meeker A.K, Isaacs W.B and Drake CG: Phenotypic analysis of prostate-infiltrating lymphocytes reveals TH17 and Treg skewing. Clin Cancer Res 14: 3254-3261, 2008.

36. Ye ZJ, Zhou Q, Gu YY, Qin SM, Ma WL, Xin JB, Tao XN and Shi HZ: Generation and differentiation of IL-17-producing $\mathrm{CD}^{+} \mathrm{T}$ cells in malignant pleural effusion. J Immunol 185: 6348-6354, 2010

37. Gnerlich JL, Mitchem JB, Weir JS, Sankpal NV, Kashiwagi H, Belt BA, Porembka MR, Herndon JM, Eberlein TJ, Goedegebuure $\mathrm{P}$ et al: Induction of Th17 cells in the tumor microenvironment improves survival in a murine model of pancreatic cancer. J Immunol 185: 4063-4071, 2010.

38. Muranski P, Boni A, Antony PA, Cassard L, Irvine KR, Kaiser A, Paulos CM, Palmer DC, Touloukian CE, Ptak K, et al: Tumor-specific Th17-polarized cells eradicate large established melanoma. Blood 112: 362-373, 2008.

39. Nigam P, Kwa S, Velu V and Amara RR: Loss of IL-17-producing CD8 $\mathrm{T}$ cells during late chronic stage of pathogenic simian immunodeficiency virus infection. J Immunol 186: 745-753, 2011.

40. Hamada H, Garcia-Hernandez ML, Reome JB, Misra SK, Strutt TM, McKinstry KK, Cooper AM, Swain SL and Dutton RW: Tc17, a unique subset of CD8 T cells that can protect against lethal influenza challenge. J Immunol 182: 3469-3481, 2009. 
41. Tajima M, Wakita D, Noguchi D, Chamoto K, Yue Z, Fugo K, Ishigame $\mathrm{H}$, Iwakura $\mathrm{Y}$, Kitamura $\mathrm{H}$ and Nishimura $\mathrm{T}$ : IL-6-dependent spontaneous proliferation is required for the induction of colitogenic IL-17-producing $\mathrm{CD} 8^{+} \mathrm{T}$ cells. J Exp Med 205: 1019-1027, 2008

42. Tzartos JS, Friese MA, Craner MJ, Palace J, Newcombe J, Esiri MM and Fugger L: Interleukin-17 production in central nervous system-infiltrating $\mathrm{T}$ cells and glial cells is associated with active disease in multiple sclerosis. Am J Pathol 172: 146-155, 2008.

43. Hinrichs CS, Kaiser A, Paulos CM, Cassard L, Sanchez-Perez L, Heemskerk B, Wrzesinski C, Borman ZA, Muranski P and Restifo NP: Type $17 \mathrm{CD}^{+} \mathrm{T}$ cells display enhanced antitumor immunity. Blood 114: 596-599, 2009.

44. Zhang Y, Hou F, Liu X, Ma D, Zhang Y, Kong B and Cui B: Tc17 cells in patients with uterine cervical cancer. PLoS One 9: e86812, 2014.

45. Zhang W, Hou F, Zhang Y, Tian Y, Jiao J, Ma D, Kong B and Cui B: Changes of Th17/Tc17 and Th17/Treg cells in endometrial carcinoma. Gynecol Oncol 132: 599-605, 2014.

46. Yu Y, Cho HI, Wang D, Kaosaard K, Anasetti C, Celis E and Yu XZ: Adoptive transfer of Tc1 or Tc17 cells elicits antitumor immunity against established melanoma through distinct mechanisms. J Immunol 190: 1873-1881, 2013.

47. Garcia-Hernandez ML, Hamada H, Reome JB, Misra SK, Tighe MP and Dutton RW: Adoptive transfer of tumor-specific Tc17 effector T cells controls the growth of B16 melanoma in mice. J Immunol 184: 4215-4227, 2010

48. Jiang G, Ma S, Wei Y, Wu Y, Yu X and Liu H: The prevalence and distribution of Th17 and Tc17 cells in patients with thyroid tumor. Immunol Lett 162: 68-73, 2014.
49. Petermann F, Rothhammer V, Claussen MC, Haas JD, Blanco LR, Heink S, Prinz I, Hemmer B, Kuchroo VK, Oukka M, et al: $\gamma \delta \mathrm{T}$ cells enhance autoimmunity by restraining regulatory $\mathrm{T}$ cell responses via an interleukin-23-dependent mechanism. Immunity 33: 351-363, 2010.

50. Sutton CE, Lalor SJ, Sweeney CM, Brereton CF, Lavelle EC and Mills KH: Interleukin-1 and IL-23 induce innate IL-17 production from gammadelta $\mathrm{T}$ cells, amplifying Th17 responses and autoimmunity. Immunity 31: 331-341, 2009.

51. Cai Y, Shen X, Ding C, Qi C, Li K, Li X, Jala VR, Zhang HG, Wang T, Zheng J, et al: Pivotal role of dermal IL-17-producing $\gamma \delta$ T cells in skin inflammation. Immunity 35: 596-610, 2011.

52. Ito $\mathrm{Y}$, Usui $\mathrm{T}$, Kobayashi $\mathrm{S}$, Iguchi-Hashimoto $\mathrm{M}$, Ito $\mathrm{H}$, Yoshitomi H, Nakamura T, Shimizu M, Kawabata D, Yukawa N, et al: Gamma/delta $\mathrm{T}$ cells are the predominant source of interleukin-17 in affected joints in collagen-induced arthritis, but not in rheumatoid arthritis. Arthritis Rheum 60: 2294-2303, 2009

53. Wakita D, Sumida K, Iwakura Y, Nishikawa H, Ohkuri T, Chamoto K, Kitamura $\mathrm{H}$ and Nishimura T: Tumor-infiltrating IL-17-producing gammadelta $\mathrm{T}$ cells support the progression of tumor by promoting angiogenesis. Eur J Immunol 40: 1927-1937, 2010.

54. Miossec P: IL-17 and Th17 cells in human inflammatory diseases. Microbes Infect 11: 625-630, 2009.

55. Tesmer LA, Lundy SK, Sarkar S and Fox DA: Th17 cells in human disease. Immunol Rev 223: 87-113, 2008.

56. Korn T, Bettelli E, Oukka M and Kuchroo VK: IL-17 and Th17 cells. Annu Rev Immunol 27: 485-517, 2009. 